\title{
Evaluation of the performance of Dutch Lipid Clinic Network score in an Italian FH population: The LIPIGEN study
}

Manuela Casula $^{\mathrm{a}, \mathrm{b}}$, Elena Olmastroni ${ }^{\mathrm{a}, \mathrm{b}}$, Angela Pirillo ${ }^{\mathrm{c}, \mathrm{d}}$, Alberico Luigi Catapano ${ }^{\mathrm{b}, \mathrm{d}, *}$, on behalf of the MEMBERS OF THE LIPIGEN STEERING COMMETTEE (Marcello Arca ${ }^{\mathrm{e}}$, Maurizio Averna ${ }^{\mathrm{f}}$, Stefano Bertolini ${ }^{g}$, Sebastiano Calandra ${ }^{\text {h }}$, Alberico Luigi Catapano ${ }^{i}$, Patrizia Tarugi ${ }^{\text {h }}$ ), PRINCIPAL INVESTIGATORS: Coordinator center (Fabio Pellegatta ${ }^{j}$ ), Participant Centers (Francesco Angelico ${ }^{\mathrm{e}}$, Marcello Arca ${ }^{\mathrm{e}}$, Maurizio Averna ${ }^{\mathrm{f}}$, Andrea Bartuli ${ }^{\mathrm{k}}$, Giacomo Biasucci ${ }^{1}$, Gianni Biolo ${ }^{\mathrm{m}}$, Luca Bonanni ${ }^{\mathrm{n}}$, Katia Bonomo ${ }^{\circ}$, Claudio Borghi ${ }^{\mathrm{p}}$, Antonio Carlo Bossi ${ }^{\mathrm{q}}$, Adriana Branchi ${ }^{\mathrm{r}}$, Francesca Carubbi ${ }^{\mathrm{s}}$, Francesco Cipollone ${ }^{\mathrm{t}}$, Nadia Citroni ${ }^{\mathrm{u}}$, Massimo Federici ${ }^{\mathrm{v}}$, Claudio Ferri $^{\mathrm{w}}$, Anna Maria Fiorenza ${ }^{\mathrm{x}}$, Andrea Giaccari ${ }^{\mathrm{y}}$, Francesco Giorgino ${ }^{\mathrm{z}}$, Ornella Guardamagna $^{\mathrm{aa}}$, Arcangelo Iannuzzi ${ }^{\mathrm{ab}}$, Lorenzo Iughetti ${ }^{\mathrm{ac}}$, Graziana Lupattelli ${ }^{\mathrm{ad}}$, Alessandro Lupi $^{\text {ae }}$, Giuseppe Mandraffino ${ }^{\text {af }}$, Rossella Marcucci ${ }^{\text {ag }}$, Lorenzo Maroni ${ }^{\text {ah }}$, Roberto Miccoli $^{\text {ai }}$, Giuliana Mombelli ${ }^{\text {aj }}$, Sandro Muntoni ${ }^{\text {ak }}$, Valerio Pecchioli ${ }^{\text {al }}$, Cristina Pederiva ${ }^{a m}$, Antonio Pipolo ${ }^{\text {an }}$, Livia Pisciotta ${ }^{a}$, Arturo Pujia ${ }^{a p}$, Francesco Purrello ${ }^{a q}$, Elena Repetti $^{\text {ar }}$, Paolo Rubba ${ }^{\text {as }}$, Carlo Sabbà ${ }^{\text {at }}$, Tiziana Sampietro ${ }^{\text {au }}$, Riccardo Sarzani ${ }^{\text {av }}$, Milena Paola Tagliabue ${ }^{\text {aw }}$, Chiara Trenti ${ }^{\text {ax }}$, Giovanni Battista Vigna ${ }^{\text {ay }}$, Josè Pablo Werba ${ }^{\text {az }}$, Sabina Zambon ${ }^{\text {ba }}$, Maria Grazia Zenti ${ }^{\text {bb }}$ ), Participant Laboratories (Ilenia Minicocci ${ }^{\mathrm{e}}$, Davide Noto $^{\mathrm{f}}$, Stefano Bertolini ${ }^{\mathrm{g}}$, Sebastiano Calandra ${ }^{\mathrm{h}}$, Giuliana Fortunato ${ }^{\mathrm{bc}}$ ), COLLABORATORS (Giuseppe Banderali ${ }^{\mathrm{am}}$, Andrea Benso ${ }^{\mathrm{aw}}{ }$, Paola Bigolin $^{\text {ba }}$, Enzo Bonora ${ }^{\text {bb }}$, Patrizia Bruzzi $^{\text {ac }}$, Marco Bucci ${ }^{\mathrm{t}}$, Paola Sabrina Buonuomo ${ }^{\mathrm{k}}$, Maria Elena Capra ${ }^{\mathrm{l}}$, Iris Cardolini ${ }^{\mathrm{v}}$, Baldassarre Cefalù ${ }^{\mathrm{f}}$, Nazzareno Cervelli ${ }^{\mathrm{w}}$, Giuseppe Chiariello ${ }^{\mathrm{ab}}$, Guido Cocci ${ }^{\mathrm{av}}$, Emanuela Colombo $^{\mathrm{x}}$, Anna Laura Cremonini ${ }^{\mathrm{ao}}$, Sergio D'Addato ${ }^{\mathrm{p}}$, Laura D'Erasmo ${ }^{\mathrm{e}}$, Beatrice Dal Pino ${ }^{\text {au }}$, Luisa De Sanctis ${ }^{\mathrm{aa}}$, Emanuele De Vita ${ }^{\mathrm{an}}$, Maria Del Ben $^{\mathrm{e}}$, Alessia Di Costanzo ${ }^{\mathrm{e}}$, Maria Donata Di Taranto ${ }^{\mathrm{bc}}$, Tommaso Fasano ${ }^{\mathrm{ax}}$, Luigi Gentile ${ }^{\mathrm{ar}}$, Marco Gentile ${ }^{\mathrm{as}}$, Omar Ghirardello ${ }^{\mathrm{ay}}$, Liliana Grigore ${ }^{\mathrm{j}}$, Milena Lussu ${ }^{\mathrm{ak}}$, Giancarla Meregalli ${ }^{\mathrm{q}}$, Simona Moffa ${ }^{\mathrm{y}}$, Tiziana Montalcini $^{\text {ap }}$, Valeria Morgia ${ }^{\text {al }}$, Fabio Nascimbeni ${ }^{\mathrm{s}}$, Andrea Pasta ${ }^{\mathrm{g}}$, Chiara Pavanello ${ }^{\mathrm{aj}}$, Antonino Saitta $^{\text {af }}$, Roberto Scicali ${ }^{\mathrm{aq}}$, Donatella Siepi ${ }^{\mathrm{ad}}$, Walter Spagnolli ${ }^{\mathrm{u}}$, Rossella Spina ${ }^{\mathrm{f}}$, Elena Sticchi $^{\text {ag }}$, Patrizia Suppressa ${ }^{\text {at }}$, Patrizia Tarugi ${ }^{\text {h }}$, Lorenzo Vigo ${ }^{\text {az }}$, Pierandrea Vinci ${ }^{\mathrm{m}}$ ), STUDY CENTRAL LABORATORY AND ANALYSIS GROUP (Alberico Luigi Catapano ${ }^{i}$, Manuela Casula $^{\text {bd }}$, Enzo Manzato ${ }^{\text {be }}$, Elena Olmastroni ${ }^{\text {bd }}$, Elena Tragni ${ }^{\text {bd }}$, Veronica Zampoleri ${ }^{\text {bf }}$ )

\footnotetext{
${ }^{a}$ Epidemiology and Preventive Pharmacology Centre (SEFAP), Department of Pharmacological and Biomolecular Sciences, University of Milan, Milan, Italy

${ }^{\mathrm{b}}$ Department of Pharmacological and Biomolecular Sciences, University of Milan, Milan, Italy

${ }^{\mathrm{c}}$ Center for the Study of Atherosclerosis, E. Bassini Hospital, Cinisello Balsamo, Milan, Italy

'IRCCS MultiMedica, Sesto S. Giovanni, Milan, Italy

e Dipartimento di Medicina Interna e Specialità Mediche "La Sapienza", A.O. Policlinico Umberto I, Rome, Italy

${ }_{\mathrm{f}}^{\mathrm{f}}$ Dipartimento Biomedico di Medicina Interna e Specialistica, Università di Palermo, Palermo, Italy

${ }^{g}$ Centro Ambulatorio Dislipidemie, U.O. Clinica di Medicina Interna 1, O. Universitaria San Martino, Genua, Italy

${ }^{\mathrm{h}}$ Laboratorio Sequenziamento Genomico, Dipartimento di Scienze Biomediche, Università di Modena e Reggio Emilia, Modena, Italy

${ }^{\mathrm{i}}$ Dipartimento di Scienze Farmacologiche e Biomolecolari, Università Degli Studi di Milano, IRCCS Multimedica, Milan, Italy
}

\footnotetext{
${ }^{*}$ Corresponding author. Department of Pharmacological and Biomolecular Sciences, University of Milan and IRCCS Multimedica, Via Balzaretti, 9, 20133, Milan, Italy.

E-mail address: alberico.catapano@unimi.it (A.L. Catapano).
} 
${ }^{j}$ Centro per Lo Studio Dell'Aterosclerosi, IRCCS Multimedica, Sesto San Giovanni, Italy

e Dipartimento di Medicina Interna e Specialità Mediche "La Sapienza", A.O. Policlinico Umberto I, Rome, Italy

${ }_{\mathrm{f}}^{\mathrm{f}}$ Dipartimento Biomedico di Medicina Interna e Specialistica, Università di Palermo, Palermo, Italy

${ }^{\mathrm{k}}$ Ambulatorio Polispecialistico per le Malattie Rare, IRCCS Ospedale Pediatrico Bambino Gesù, Rome, Italy

${ }^{1}$ Centro Dislipidemie in Età Evolutiva U.O. Pediatria e Neonatologia, Ospedale G. da Saliceto, Piacenza, Italy

${ }^{\mathrm{m}}$ S.S. Diabetologia e Malattie Metaboliche, U.C.O. Clinica Medica Generale, Azienda Ospedaliera Universitaria OORR, Ospedale Maggiore, Trieste, Italy

${ }^{\mathrm{n}}$ Ambulatorio Dislipidemie, UO Medicina Interna, Ospedale Dell'Angelo di Mestre, Venice, Italy

${ }^{\circ}$ AOU San Luigi Gonzaga, Orbassano, Turin, Italy

${ }^{\mathrm{P}}$ U.O. di Medicina Interna, Centro Aterosclerosi, Ambulatorio Dislipidemie, Ospedale Policlinico S. Orsola-Malpighi, Bologna, Italy

${ }^{\mathrm{q}}$ U.O.C. Malattie Endocrine e Centro Regionale per Il Diabete (Diabetologia), Ospedale "Treviglio-Caravaggio" di Treviglio, Bergamo, Italy

${ }^{\mathrm{r}}$ Ambulatorio Dislipidemie, Centro per Lo Studio e La Prevenzione Dell'Arteriosclerosi, Fondazione IRCCS Ca' Granda, Ospedale Maggiore Policlinico e Dipartimento di

Scienze Cliniche e di Comunità, Università Degli Studi di Milano, Milan, Italy

${ }^{\mathrm{s}}$ U.O. Medicina Ad Indirizzo Metabolico-nutrizionistico, Centro Dislipidemie e Centro di Riferimento Regionale per le Malattie Metaboliche Rare, Nuovo Ospedale S.

Agostino Estense (NOCSAE), Modena, Italy

${ }^{\mathrm{t}}$ Centro di Alta Specializzazione per La Prevenzione Dell'arteriosclerosi, Centro di Eccellenza ESH per L'ipertensione Arteriosa, Centro di Riferimento Regionale per le

Dislipemie, Ospedale Policlino S.S. Annunziata, Chieti, Italy

${ }^{u}$ Centro Dislipidemia, UO Medicina Interna, Ospedale Santa Chiara, Trento, Italy

v Dipartimento Medicina Interna, Centro per L'Aterosclerosi, Policlinico Universtario “Tor Vergata”, Rome, Italy

${ }^{\mathrm{w}}$ Centro Ipertensione Arteriosa e Prevenzione Cardiovascolare UOC Medicina Interna e Nefrologia, L'Aquila, Italy

${ }^{x}$ Dip. Medicina Interna, Centro Prevenzione e Cura Dell'aterosclerosi, A.O. "Guido Salvini”, Garbagnate Milanese, Milan, Italy

${ }^{\mathrm{y}}$ UOC Endocrinologia e Malattie Del Metabolismo, Policlinico Gemelli, Rome, Italy

${ }^{\text {z } U . O . ~ E n d o c r i n o l o g i a, ~ A m b u l a t o r i ~ d i ~ D i a b e t o l o g i a ~ e ~ M a l a t t i e ~ M e t a b o l i c h e, ~ A . O . ~ U n i v e r s i t a r i a ~ P o l i c l i n i c o ~ C o n s o r z i a l e, ~ U n i v e r s i t a ̀ ~ D e g l i ~ S t u d i ~ d i ~ B a r i ~ " A l d o ~ M o r o ", ~ B a r i, ~ I t a l y ~}$

${ }^{\text {aa }}$ U.O. Dislipidemie e Prevenzione Cardiovascolare, Ospedale Regina Margherita, Turin, Italy

${ }^{\text {ab }}$ U.O. Medicina Interna 5, Centro per le Malattie da Arteriosclerosi, AORN Cardarelli, Naples, Italy

${ }^{\text {ac }}$ U.O. Clinica Pediatrica, Policlinico di Modena, Modena, Italy

${ }^{\text {ad }}$ U.O. Medicina Interna Angiologia, Malattie da Arteriosclerosi, Ambulatorio di Malattie Del Ricambio Lipidico, Ospedale Santa Maria Della Misericordia, Perugia, Italy

${ }^{\text {ae }}$ ASL VCO, UO SOC Cardiologia, Ospedale Castelli, Verbania, Italy

${ }^{\text {af }}$ Dipartimento di Medicina Interna e Terapia Medica, Centro per La Diagnosi e Cura Della Dislipidemia e Prevenzione Dell'Aterosclerosi, A.O. Universitaria Policlinico “G.

Martino", Messina, Italy

${ }^{a g}$ Ambulatorio Malattie Aterotrombotiche, AOUC Azienda Ospedaliero-Universitaria Careggi, Florence, Italy

${ }^{\text {ah }}$ Ambulatorio Ipertensione Dislipidemie, U.O. Medicina Generale, ASST Valle Olona, Ospedale di Gallarate, Gallarate, Italy

${ }^{\text {ai }}$ U.O. Diabetologia e Malattie Metaboliche, Centro per La Prevenzione e La Terapia Delle Dislipidemie e Dell'aterosclerosi, A.O.U. Pisana Ospedale Cisanello, Pisa, Italy

${ }^{\text {aj }}$ Centro Universitario Dislipidemie "E. Grossi Paoletti", A.O. Ospedale Niguarda Ca' Granda, Milan, Italy

${ }^{\text {ak }}$ Centro per le Malattie Dismetaboliche e L'arteriosclerosi, Associazione ME.DI.CO Onlus, Cagliari, Italy

${ }^{\text {al } U O S D ~ ' P r e v e n z i o n e ~ C a r d i o v a s c o l a r e ', ~ D i p a r t i m e n t o ~ d i ~ S c i e n z e ~ M e d i c h e, ~ A z i e n d a ~ S a n i t a r i a ~ L o c a l e ~ F r o s i n o n e, ~ F r o s i n o n e, ~ I t a l y ~}$

${ }^{a m}$ U.O. Clinica Pediatrica, Servizio Clinico Dislipidemie per Lo Studio e La Prevenzione Dell'Aterosclerosi in Età Pediatrica, Ospedale San Paolo, Milan, Italy

${ }^{\text {an }}$ AOU San Giovanni di Dio e Ruggi D'Aragona, Salerno, Italy

${ }^{\text {ao }}$ U.O. Clinica di Medicina Interna 1, Ambulatorio Dislipidemie, IRCCS, A.O.U. San Martino, IST, Genoa, Italy

ap A.O.U. Mater Domini, Catanzaro, UOC di Nutrizione Clinica, Ambulatorio Dislipidemie, Catanzaro, Italy

${ }^{\text {aq }}$ U.O. Medicina Interna, Ospedale "Garibaldi Nesima”, Catania, Italy

${ }^{\mathrm{ar}}$ Società di Diabetologia e Malattie Metaboliche, Asti, Italy

${ }^{\text {as } C e n t r o ~ C o o r d i n a m e n t o ~ R e g i o n a l e ~ p e r ~ l e ~ I p e r l i p i d e m i e, ~ A O U ~ P o l i c l i n i c o ~ F e d e r i c o ~ I I, ~ N a p l e s, ~ I t a l y ~}$

at U.O. di Medicina Interna "Frugoni" e Centro di Assistenza e Ricerca Malattie Rare, A.O. Universitaria Policlinico Consorziale, Università Degli Studi di Bari "Aldo Moro", Bari, Italy

${ }^{a}$ U.O. Lipoaferesi, Centro Regionale di Riferimento per La Diagnosi e Cura Delle Dislipidemie Ereditarie, Fondazione Toscana "G. Monasterio", Pisa, Italy

${ }^{\text {av }}$ Clinica di Medicina Interna e Geriatria, Centro di Riferimento Regionale Ipertensione Arteriosa e Malattie Cardiovascolari, INRCA Ospedale "Sestilli" e Azienda

Ospedaliero-Universitaria Ospedali Riuniti di Torrette di Ancona, Ancona, Italy

${ }^{\mathrm{aw}}$ SCDU Endocrinologia, Diabetologia e Metabolismo, Dipartimento di Scienze Mediche, Università di Torino, Turin, Italy

${ }^{\mathrm{ax}}$ Arcispedale S. Maria Nuova, Azienda Ospedaliera di Reggio Emilia, Reggio Emilia, Italy

${ }^{\text {ay }}$ U.O Medicina Interna Universitaria, Centro per Lo Studio Delle Dislipidemie e Dell'Aterosclerosi Azienda Ospedaliero-Universitaria di Ferrara, Polo di Cona, Ferrara, Italy

${ }^{\mathrm{az}}$ U.O. Ambulatorio Prevenzione Aterosclerosi IRCCS Cardiologico Monzino, Milan, Italy

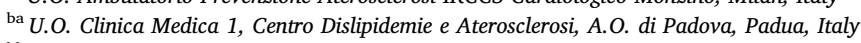

${ }^{\mathrm{b}}$ U.O. Endocrinologia, Diabetologia e Malattie Del Metabolismo, Centro Regionale Specializzato per La Diagnosi e Terapia Delle Dislipidemie e Aferesi Terapeutica, A.O.

Universitaria Integrata di Verona, Verona, Italy

e Dipartimento di Medicina Interna e Specialità Mediche "La Sapienza", A.O. Policlinico Umberto I, Rome, Italy

${ }_{\mathrm{f}}^{\mathrm{f}}$ Dipartimento Biomedico di Medicina Interna e Specialistica, Università di Palermo, Palermo, Italy

${ }^{g}$ Centro Ambulatorio Dislipidemie, U.O. Clinica di Medicina Interna 1, O. Universitaria San Martino, Genua, Italy

${ }^{\mathrm{h}}$ Laboratorio Sequenziamento Genomico, Dipartimento di Scienze Biomediche, Università di Modena e Reggio Emilia, Modena, Italy

${ }^{\mathrm{bc}}$ Laboratorio di Screening di Malattie Metaboliche, CEINGE - Biotecnologie Avanzate, Dipartimento di Biochimica e Biotecnologie Mediche, Azienda Ospedaliera

Universitaria "Federico II", Naples, Italy

e Dipartimento di Medicina Interna e Specialità Mediche "La Sapienza", A.O. Policlinico Umberto I, Rome, Italy

${ }_{\mathrm{f}}^{\mathrm{f}}$ Dipartimento Biomedico di Medicina Interna e Specialistica, Università di Palermo, Palermo, Italy

${ }^{\mathrm{g}}$ Centro Ambulatorio Dislipidemie, U.O. Clinica di Medicina Interna 1, O. Universitaria San Martino, Genua, Italy

${ }^{\mathrm{h}}$ Laboratorio Sequenziamento Genomico, Dipartimento di Scienze Biomediche, Università di Modena e Reggio Emilia, Modena, Italy

${ }^{\mathrm{j}}$ Centro per Lo Studio Dell'Aterosclerosi, IRCCS Multimedica, Sesto San Giovanni, Italy

${ }^{\mathrm{k}}$ Ambulatorio Polispecialistico per le Malattie Rare, IRCCS Ospedale Pediatrico Bambino Gesù, Rome, Italy

${ }^{1}$ Centro Dislipidemie in Età Evolutiva U.O. Pediatria e Neonatologia, Ospedale G. da Saliceto, Piacenza, Italy

${ }^{\mathrm{m}}$ S.S. Diabetologia e Malattie Metaboliche, U.C.O. Clinica Medica Generale, Azienda Ospedaliera Universitaria OORR, Ospedale Maggiore, Trieste, Italy

${ }^{\mathrm{p}}$ U.O. di Medicina Interna, Centro Aterosclerosi, Ambulatorio Dislipidemie, Ospedale Policlinico S. Orsola-Malpighi, Bologna, Italy

${ }^{\mathrm{q}}$ U.O.C. Malattie Endocrine e Centro Regionale per Il Diabete (Diabetologia), Ospedale "Treviglio-Caravaggio" di Treviglio, Bergamo, Italy

${ }^{\mathrm{s}}$ U.O. Medicina Ad Indirizzo Metabolico-nutrizionistico, Centro Dislipidemie e Centro di Riferimento Regionale per le Malattie Metaboliche Rare, Nuovo Ospedale S. Agostino Estense (NOCSAE), Modena, Italy

${ }^{\mathrm{t}}$ Centro di Alta Specializzazione per La Prevenzione Dell'arteriosclerosi, Centro di Eccellenza ESH per L'ipertensione Arteriosa, Centro di Riferimento Regionale per le Dislipemie, Ospedale Policlino S.S. Annunziata, Chieti, Italy

${ }^{\mathrm{u}}$ Centro Dislipidemia, UO Medicina Interna, Ospedale Santa Chiara, Trento, Italy

${ }^{\vee}$ Dipartimento Medicina Interna, Centro per L'Aterosclerosi, Policlinico Universtario “Tor Vergata”, Rome, Italy

${ }^{\mathrm{w}}$ Centro Ipertensione Arteriosa e Prevenzione Cardiovascolare UOC Medicina Interna e Nefrologia, L'Aquila, Italy

${ }^{x}$ Dip. Medicina Interna, Centro Prevenzione e Cura Dell'aterosclerosi, A.O. "Guido Salvini”, Garbagnate Milanese, Milan, Italy

${ }^{y}$ UOC Endocrinologia e Malattie Del Metabolismo, Policlinico Gemelli, Rome, Italy

${ }^{\text {aa }}$ U.O. Dislipidemie e Prevenzione Cardiovascolare, Ospedale Regina Margherita, Turin, Italy

${ }^{\mathrm{ab}}$ U.O. Medicina Interna 5, Centro per le Malattie da Arteriosclerosi, AORN Cardarelli, Naples, Italy 
${ }^{\text {ac }}$ U.O. Clinica Pediatrica, Policlinico di Modena, Modena, Italy

${ }^{\text {ad }}$ U.O. Medicina Interna Angiologia, Malattie da Arteriosclerosi, Ambulatorio di Malattie Del Ricambio Lipidico, Ospedale Santa Maria Della Misericordia, Perugia, Italy

${ }^{\text {af }}$ Dipartimento di Medicina Interna e Terapia Medica, Centro per La Diagnosi e Cura Della Dislipidemia e Prevenzione Dell'Aterosclerosi, A.O. Universitaria Policlinico “G.

Martino", Messina, Italy

${ }^{\text {ag }}$ Ambulatorio Malattie Aterotrombotiche, AOUC Azienda Ospedaliero-Universitaria Careggi, Florence, Italy

${ }^{\text {aj }}$ Centro Universitario Dislipidemie "E. Grossi Paoletti", A.O. Ospedale Niguarda Ca' Granda, Milan, Italy

${ }^{\mathrm{ak}}$ Centro per le Malattie Dismetaboliche e L'arteriosclerosi, Associazione ME.DI.CO Onlus, Cagliari, Italy

${ }^{\text {al }}$ UOSD 'Prevenzione Cardiovascolare', Dipartimento di Scienze Mediche, Azienda Sanitaria Locale Frosinone, Frosinone, Italy

${ }^{a m}$ U.O. Clinica Pediatrica, Servizio Clinico Dislipidemie per Lo Studio e La Prevenzione Dell'Aterosclerosi in Età Pediatrica, Ospedale San Paolo, Milan, Italy

an AOU San Giovanni di Dio e Ruggi D'Aragona, Salerno, Italy

${ }^{\text {ao }}$ U.O. Clinica di Medicina Interna 1, Ambulatorio Dislipidemie, IRCCS, A.O.U. San Martino, IST, Genoa, Italy

${ }^{a p}$ A.O.U. Mater Domini, Catanzaro, UOC di Nutrizione Clinica, Ambulatorio Dislipidemie, Catanzaro, Italy

${ }^{\text {aq }}$ U.O. Medicina Interna, Ospedale "Garibaldi Nesima", Catania, Italy

${ }^{\text {ar }}$ Società di Diabetologia e Malattie Metaboliche, Asti, Italy

${ }^{\text {as }}$ Centro Coordinamento Regionale per le Iperlipidemie, AOU Policlinico Federico II, Naples, Italy

${ }^{\text {at }}$ U.O. di Medicina Interna "Frugoni" e Centro di Assistenza e Ricerca Malattie Rare, A.O. Universitaria Policlinico Consorziale, Università Degli Studi di Bari "Aldo Moro", Bari, Italy

${ }^{\text {au }}$ U.O. Lipoaferesi, Centro Regionale di Riferimento per La Diagnosi e Cura Delle Dislipidemie Ereditarie, Fondazione Toscana "G. Monasterio", Pisa, Italy

${ }^{a v}$ Clinica di Medicina Interna e Geriatria, Centro di Riferimento Regionale Ipertensione Arteriosa e Malattie Cardiovascolari, INRCA Ospedale "Sestilli" e Azienda

Ospedaliero-Universitaria Ospedali Riuniti di Torrette di Ancona, Ancona, Italy

${ }^{a w}$ SCDU Endocrinologia, Diabetologia e Metabolismo, Dipartimento di Scienze Mediche, Università di Torino, Turin, Italy

${ }^{a x}$ Arcispedale S. Maria Nuova, Azienda Ospedaliera di Reggio Emilia, Reggio Emilia, Italy

${ }^{\text {ay }}$ U.O Medicina Interna Universitaria, Centro per Lo Studio Delle Dislipidemie e Dell'Aterosclerosi Azienda Ospedaliero-Universitaria di Ferrara, Polo di Cona, Ferrara, Italy

${ }^{a}$ U.O. Ambulatorio Prevenzione Aterosclerosi IRCCS Cardiologico Monzino, Milan, Italy

${ }^{\text {ba }}$ U.O. Clinica Medica 1, Centro Dislipidemie e Aterosclerosi, A.O. di Padova, Padua, Italy

${ }^{\mathrm{b}}$ U.O. Endocrinologia, Diabetologia e Malattie Del Metabolismo, Centro Regionale Specializzato per La Diagnosi e Terapia Delle Dislipidemie e Aferesi Terapeutica, A.O.

Universitaria Integrata di Verona, Verona, Italy

${ }^{\mathrm{bc}}$ Laboratorio di Screening di Malattie Metaboliche, CEINGE - Biotecnologie Avanzate, Dipartimento di Biochimica e Biotecnologie Mediche, Azienda Ospedaliera

Universitaria "Federico II", Naples, Italy

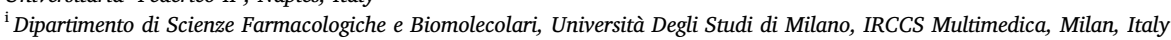

${ }^{b d}$ Centro Universitario di Epidemiologia e Farmacologia Preventiva (SEFAP), Dipartimento di Science Farmacologiche e Biomolecolari, Università Degli Studi di Milano, Milan, Italy

be Dipartimento di Medicina (DIMED), Sezione Geriatrica, Università di Padova, Padua, Italy

${ }^{\text {bf }}$ Centro per Lo Studio Dell'Aterosclerosi, Ospedale E. Bassini, Cinisello Balsamo, Milan, Italy

\section{H I G H L I G H T S}

- FH is a genetic disorder characterized by high levels of cholesterol from birth.

- Identification of FH subjects is crucial to prevent premature cardiovascular events.

- FH is clinically diagnosed by means of several criteria, including the DLCN score.

- The application of the DLCN score may be limited by missing patient information.

\section{A R T I C L E I N F O}

\section{Keywords:}

Familial hypercholesterolemia

Dutch Lipid Clinic Network score

Genetic testing

\begin{abstract}
A B S T R A C T
Background and aims: Familial hypercholesterolemia (FH) is an inherited disorder characterized by high levels of blood cholesterol from birth and premature coronary heart disease. Thus, the identification of FH patients is crucial to prevent or delay the onset of cardiovascular events, and the availability of a tool helping with the diagnosis in the setting of general medicine is essential to improve FH patient identification.

Methods: This study evaluated the performance of the Dutch Lipid Clinic Network (DLCN) score in FH patients enrolled in the LIPIGEN study, an Italian integrated network aimed at improving the identification of patients with genetic dyslipidaemias, including FH.

Results: The DLCN score was applied on a sample of 1377 adults (mean age $42.9 \pm 14.2$ years) with genetic diagnosis of $\mathrm{FH}$, resulting in $28.5 \%$ of the sample classified as probable $\mathrm{FH}$ and $37.9 \%$ as classified definite $\mathrm{FH}$. Among these subjects, $43.4 \%$ had at least one missing data out of 8 , and about $10.0 \%$ had 4 missing data or more. When analyzed based on the type of missing data, a higher percentage of subjects with at least 1 missing data in the clinical history or physical examination was classified as possible FH (DLCN score 3-5). We also found that using real or estimated pre-treatment LDL-C levels may significantly modify the DLCN score. Conclusions: Although the DLCN score is a useful tool for physicians in the diagnosis of FH, it may be limited by the complexity to retrieve all the essential information, suggesting a crucial role of the clinical judgement in the identification of FH subjects.
\end{abstract}

\section{Introduction}

Familial hypercholesterolaemia (FH) is a monogenic disorder characterized by increased LDL cholesterol (LDL-C) levels from birth and increased risk of early coronary heart disease (CHD) [1]. The early identification of FH subjects is therefore essential to reduce the burden of cholesterol and prevent or at least delay the occurrence of cardiovascular events. The early initiation of lipid-lowering therapies in these subjects will reduce morbidity and mortality for premature CHD, and will also have an economical return. Unfortunately, $\mathrm{FH}$ is an underdiagnosed condition and, as a consequence, commonly undertreated until the occurrence of the first cardiovascular event [1].

The diagnosis of FH may be achieved either by targeted screening, aimed at identifying FH cases among hypercholesterolemic subjects with personal or family history of premature CHD or hypercholesterolemia, or, in alternative, by cascade screening (including genetic testing), aimed at identifying first- and second-degree family members of a subject diagnosed with $\mathrm{FH}$. The targeted screening approach is cost-effective, but entails the risk of missing $30-60 \%$ of affected patients; the cascade screening approach guarantees a higher detection rate, although a considerable risk of missing affected individuals is still present. These observations have prompted some of the recent guidelines to recommend a strategy of universal lipid screening of 
children [1] However, the cost effectiveness and utility of universal screening are still undefined. Furthermore, a minority of $\mathrm{FH}$ patients may have a normal lipid profile at the time of screening, thus facing the risk of missing the diagnosis in some people despite screening of the entire population.

In the context of familial hypercholesterolemia, a relevant question is what to screen - lipids or genes? Genetic screening strategy involves searching for mutations in the common genes causing FH among suspected subjects and, possibly, their close relatives. It is worth noting that a relevant proportion (20-40\%) of individuals clinically diagnosed with $\mathrm{FH}$ does not present a causative mutation in any of the conventionally tested genes. This observation, together with the results of genomewide association studies, showing that the simultaneous presence of a number of single nucleotide polymorphisms may significantly influence LDL-C levels (polygenic hypercholesterolemia), suggests the possible involvement of variants in multiple genes, each of which has a small effect but when in association may increase LDL-C levels at the typical range observed in patients with monogenic $\mathrm{FH}$ [2]. In such patients, genetic cascade testing is expected to have a very low yield and is unlikely to be cost-effective. Hence, genetic cascade screening is likely to benefit only probands where a definite mutation is identified; in others, a strategy of lipid profilebased cascade screening may be preferable.

$\mathrm{FH}$ is clinically diagnosed on the basis of clinical characteristics and laboratory parameters; criteria to identify FH subjects include the MEDPED (Make Early Diagnosis to Prevent Early Deaths) score [3] and the Simon Broome criteria [4], based on the LDL-C values and the family clinical history, and the Dutch Lipid Clinic Network (DLCN) score, which also includes physical characteristics such as tendon xanthomas [5] (Supplementary table 1). The availability of a tool that guides diagnosis in the setting of general medicine (or for health professionals not specialized in the management of lipid metabolism diseases) is crucial to improve FH patient identification and to start the appropriate pharmacological therapy as soon as possible. However, it is not clear whether the performance of the available diagnostic scores may efficiently apply to different countries or subpopulations (e.g., age groups, mild phenotypes) and how much missing information may impact on the diagnosis rate.

In the present study, we aimed at evaluating the performance of the DLCN score in patients with genetic diagnosis of FH enrolled in the LIPIGEN (LIpid TransPort Disorders Italian GEnetic Network) network [6], addressing the question whether missing information may affect the identification of $\mathrm{FH}$ subjects.

\section{Materials and methods}

The LIPIGEN is an integrated network aimed at improving the identification of patients with genetic dyslipidaemias, including FH, in Italy [6]. The LIPIGEN-FH study, an observational, multicenter, retrospective and prospective study started in 2012 [6], collects data on FH patients followed by lipid clinics all over Italy as part of the normal clinical practice. Available information includes demographic and clinical data (age, gender, personal and family history of hypercholesterolemia or premature cardiovascular or cerebrovascular events, data from physical examination), pharmacological therapies and biochemical data. After the visit by a specialized physician, patients with clinical suspect of primary hypercholesterolemia are referred for genetic testing of the appropriate candidate genes. The decision to address a subject to the genetic testing may be based either on the application of the clinical score or on the decision of the lipid specialist, supported by anomalies in her/his lipid profile or by the presence of a familial history of premature cardiovascular disease (even in the absence of individual increased LDL-C levels, as for example in children). The identification of a causative mutation in a patient is then followed by the cascade screening of family members to identify new cases of $\mathrm{FH}$, who undergo genetic testing if $\mathrm{FH}$ is clinically suspected.

To test the performance of the DLCN score, the analysis was carried out in all mutation-positive patients (as established by genetic test performed in different laboratories), aged 18 years or more, who underwent clinical evaluation and had available information on LDL-C levels. The population used for this analysis included both FH index cases and the FH relatives identified by cascade screening. The DLCN score performance was evaluated also as a function of the number and type of missing parameters. In the absence of available pre-therapy LDL-C values (as a part of the DLCN score), they were estimated from the actual levels adjusting by correction factors which consider the type and dose of current lipid-lowering therapy [7]. As sensitivity analysis, the performance of the DLCN score was evaluated also in a smaller sample of patients $(\mathrm{N}=343)$ with clinical suspect of $\mathrm{FH}$ who have been genetic tested by a centralized laboratory searching for a broad range of possible mutations of several candidates genes. For exploratory purposes, the DLCN score was applied also on patients aged less than 18 years, in whom the algorithm has not been validated.

Continuous variables are presented as mean $\pm \mathrm{SD}$, whereas categorical variables are presented as cases (n) and percentage rate (\%). To define the sensitivity of the DLCN score, the Bayes' theorem was applied on the subsample.

\section{Results}

A total of 1377 mutation-positive adult patients has been included in the present analysis. Supplementary table 2 provides the general characteristics of these subjects. The number of men and women in the sample was comparable (48.6\% vs $51.3 \%$ respectively), mean BMI value was $25.5( \pm 4.4) \mathrm{Kg} / \mathrm{m}^{2}$, mean glucose level was $90.1( \pm 18.2) \mathrm{mg} / \mathrm{dL}$. Mean LDL-C, HDL-C, TG levels were 285.5 ( \pm 95.0$) \mathrm{mg} / \mathrm{dL}, 52.8( \pm 14.3) \mathrm{mg} / \mathrm{dL}$, and $121.4( \pm 67.4) \mathrm{mg} /$ $\mathrm{dL}$, respectively. Among the subjects included in the analysis, $44.2 \%$ were on statin therapy.

When applied to this population with positive genetic test, the DLCN score classified as probable FH (score 6-8) $28.5 \%$ and as definite FH (score $\geq 9$ ) $37.9 \%$ of subjects; $66.4 \%$ had thus a DLCN score $\geq 6$ and defined as potential FH (Fig. 1). Similar results were observed when the DLCN score was applied to the subgroup who underwent the genetic testing in a centralized laboratory

\section{FH diagnosis with DLCN score}

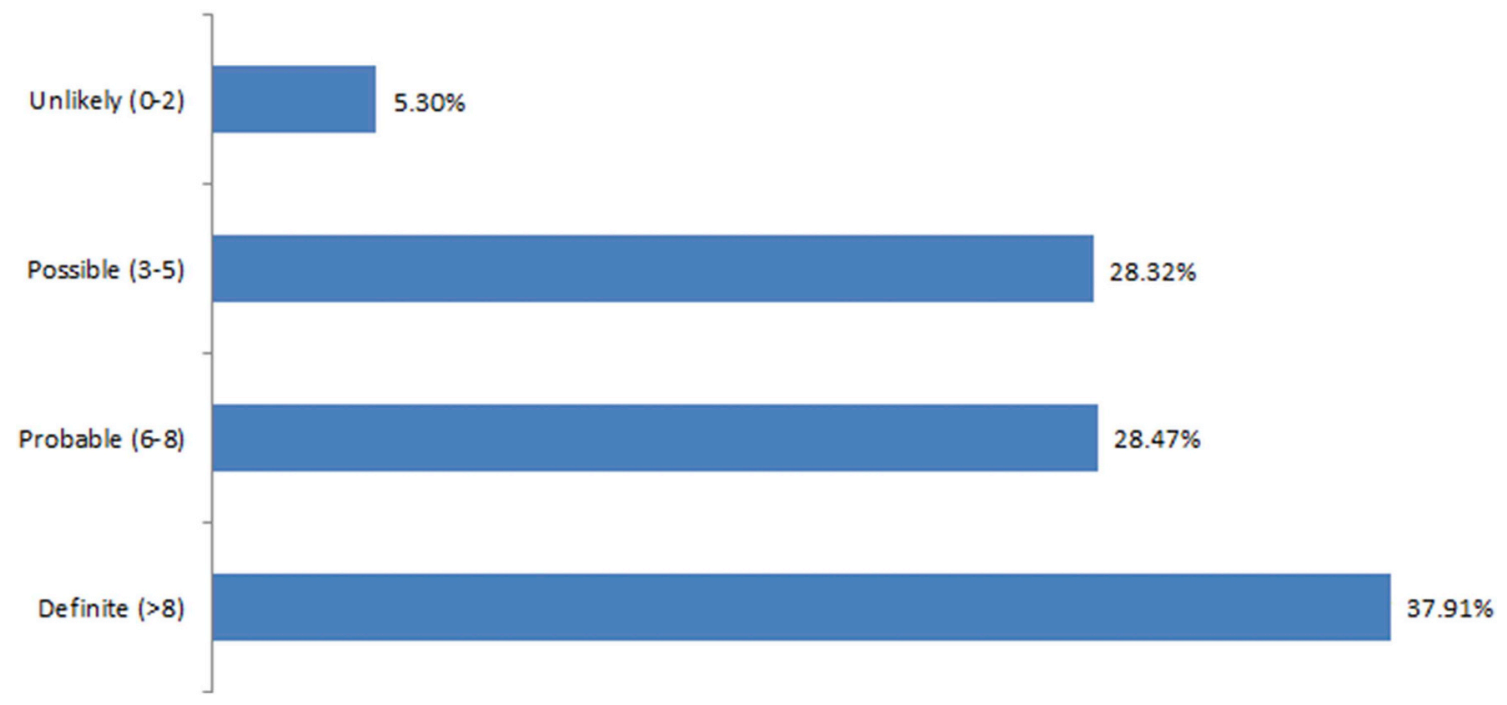

Fig. 1. DLCN score in the mutation-positive group of the LIPIGEN Study. 
Table 1

Number of missing information.

\begin{tabular}{llll}
\hline \multirow{2}{*}{$\begin{array}{l}\text { Number of missing } \\
\text { information }\end{array}$} & \multicolumn{2}{l}{ Distribution } \\
\cline { 2 - 4 } & Frequency & Percentages & $\begin{array}{l}\text { Cumulative } \\
\text { percentages }\end{array}$ \\
\hline 0 & & & 56.57 \\
1 & 779 & 56.57 & 71.75 \\
2 & 209 & 15.18 & 85.19 \\
3 & 185 & 13.44 & 90.05 \\
$\geq 4$ & 67 & 4.87 & 100.00 \\
& 137 & 9.95 &
\end{tabular}

Table 2

Missing criteria.

\begin{tabular}{ll}
\hline Criteria & Missing (\%) \\
\hline First-degree relative with known premature CHD & 11.62 \\
First-degree relative with known LDL cholesterol > 95th percentile & 12.85 \\
First-degree relative with tendon xanthoma and/or corneal arcus & 34.57 \\
Child(ren) < 18 years with LDL cholesterol > 95th percentile & 25.78 \\
Subject has premature CHD & 9.08 \\
Subject has premature cerebral or peripheral vascular disease & 10.17 \\
Tendon xanthoma & 5.37 \\
Corneal arcus in a person $<45$ years & 11.62 \\
\hline
\end{tabular}

( $29.2 \%$ and $34.9 \%$, respectively). The presence of variants of uncertain clinical significance was $11.0 \%$ (DLCN score $\geq 9$ ), $21.3 \%$ (DLCN score 6-8), 31.3\% (DLCN score 3-5), and 45.5\% (DLCN score 0-2) (Supplementary table 3). The Bayes theorem showed that the sensitivity of DLCN test is 0.33 .

Overall, in our sample, only $56.6 \%$ of patients had all the information required to calculate the DLCN score through the 8 criteria besides LDL-cholesterol, and about $10.0 \%$ had 4 or more missing data (Table 1). In particular, among subjects with a DLCN score of 5 (9.2\%), just below the threshold of the possible diagnosis, about $46.0 \%$ had at least one missing criteria information.

About $34.6 \%$ of patients had not information on the presence of tendon xanthoma and/or corneal arcus in first-degree relatives and $11.6 \%$ and $12.9 \%$ had not information on positive history of premature coronary heart disease (CHD) or hypercholesterolemia in first-degree relatives, respectively. The information on premature CHD or on cerebral/peripheral vascular disease was missing in $9.1 \%$ and $10.2 \%$ of the subjects, respectively (Table 2 ). The nature of missing information had a differential impact on the ability of the DLCN score to identify FH patients. Thus, the lack of information related to the family clinical history did not modify the rate of patient identification compared with that of subjects without missing data (Table 3); in contrast, the lack of information concerning the physical signs typical of FH or the personal history of cardio/cerebrovascular events strongly reduced the percentage of subjects classified as definite FH (Table 3).

As for many FH subjects the pre-treatment LDL-C levels were not available, we evaluated whether the use of estimated pre-treatment LDL-C levels (adjusting by correction factors considering the type and dose of current lipid-lowering therapy) might affect the DLCN score. Patients on statin therapy (representing $44.2 \%$ of the whole studied population) were grouped based on the availability of their pretreatment LDL-C levels or not $(65.2 \%$ and $34.8 \%$ of the on-statin therapy group, respectively) and compared with subjects not on statin therapy. Within the first subgroup, $26.2 \%$ had pre-treatment LDL-C levels $>325 \mathrm{mg} / \mathrm{dL}$ (Table 4), in line with what observed in patients not on statin treatment $(22.0 \%)$; in contrast, $39.6 \%$ of subjects with estimated pre-treatment LDL-C value had LDL-C levels $\geq 325 \mathrm{mg} / \mathrm{dL}$ (Table 4). This translated into different percentages of patients classified as probable or definite FH by the DLCN score $(\geq 6)$ (Table 4).

Finally, when the DLCN score was applied in the mutation-positive paediatric population ( $<18$ years) in whom the algorithm has not been validated, the diagnosis was unlikely for $29.2 \%$ and definitive for only $7.5 \%$ of children.

\section{Discussion}

Due to the high burden of cholesterol from birth, patients with $\mathrm{FH}$ have a significantly increased risk of developing atherosclerosis early in the life and may experience premature coronary heart disease. Thus, these patients need to be aggressively and promptly treated to reduce their cardiovascular risk. Despite this awareness, FH is largely underdiagnosed in most countries [1] and frequently the diagnosis of $\mathrm{FH}$ is done following a casual biochemical evaluation of LDL-C levels or after the occurrence of a premature cardiovascular event. In addition, in most cases $\mathrm{FH}$ is undertreated, as reported in a study showing that only $48 \%$ of $\mathrm{FH}$ subjects receive statins [8], and frequently the dose of statin provided is not adequate to reduce their plasma LDL-C to the levels recommended by current guidelines [1,9]; finally, statin therapy is often introduced too late in life.

From all these considerations, it is evident that a timely diagnosis of $\mathrm{FH}$ is crucial to start immediately with a pharmacological approach integrated with lifestyle modifications, in order to reduce the overall cardiovascular risk of $\mathrm{FH}$ patients, thus gaining time free of cardiovascular events. Therefore, the availability of diagnostic tools that can be widely and easily used by physicians may represent a relevant opportunity for the identification of high cardiovascular risk patients.

From our analysis, it is clear that, despite the subjects had a positive genetic test which defined their FH condition, the "a posteriori" application of the DLCN score could not classify all of them as definite FH. Overall, less than half of subjects were classified as definite FH (37.9\%).

Due to its structure, one major limit in the application of the DLCN score is the fact that it derives not only from objective information (biochemical evaluation of LDL-C levels and physical examination), but also from the personal and family cardiovascular history, which may be more difficult to be unbiased. The weight of missing information in the determination of the final score is not clear. However, the lack of one or more parameters which are part of the algorithm may reduce the final score and may lead to the attribution of an incorrect FH category. This may be of particular relevance for those subjects having a DLCN score of 5 and one or more missing data, as they might increase their score in the presence of further positive information and thus be shifted to the probable or even definite FH category. Obviously, this may affect the possibility of addressing the subject to the genetic testing (which is strongly recommended among individuals with DLCN score $>5$ ) to determine the presence of a causative mutation and may also have an impact on the type of pharmacological approach, although this is commonly driven by LDL-C plasma levels and not by genotype. On the other hand, it appears that a large percentage of mutation-positive subjects with no missing information would have been classified as unlikely or possible FH ( $\sim 35 \%)$ by the application of the DUTCH score; despite that, the lipid specialists addressed them to the genetic testing on the basis of their specific knowledge about $\mathrm{FH}$, recognizing that it is a pathologic condition which may be present with a highly variable phenotypes and that a low DUTCH score may be not always suggestive of a negative FH condition. This means that the final decision of the lipid specialists is essential to increase the detection rate of FH.

Among subjects with at least 1 missing data, those lacking information on the personal clinical history or physical examination were more likely to be categorized in the "possible $\mathrm{FH}$ " group (54.6\% and 40.4\%, respectively). In the setting of a new diagnosis, this could lead to an underestimation of the individual risk to have $\mathrm{FH}$, and thus may induce the general practitioner not to investigate further this possibility and therefore the subject would not be directed to the genetic test. Indeed, although the genetic test to detect an underlying molecular defect in an index FH patient is costly, it allows early diagnosis, even in childhood, and is carried out once in a lifetime; in addition, the FH genetic diagnosis provides a cost-effective tool for cascade testing of the FH index case relatives and to prevent premature CHD. As a consequence, also the therapeutic strategy adopted might be inadequate to treat this type of patient. Based on these considerations, it is evident that the appropriate diagnosis can have a relevant clinical impact.

Another critical issue concerns the LDL-C levels, as the score should be applied using pre-therapy values, while many of the available LDL-C level values are obtained post-statin therapy, and thus need to be adjusted based on the drug type and dose. However, this could lead to an overestimation of the pre-treatment LDL-C levels [10]. In our study, we found that, in the group with estimated pre-statin LDL-C levels, a higher percentage of subjects had values $>325 \mathrm{mg} / \mathrm{dL}$, which, by conferring the highest score for this category (Supplementary table 1), translated into a higher percentage of subjects categorized as definite $\mathrm{FH}$ compared with the group having measured pre-statin LDL-C levels.

It is possible that the DLCN score needs adaptations when applied to populations other than the original one. Even more, clinicians should remember that this tool has not been developed for the paediatric population. Indeed, when applied to the LIPIGEN paediatric population with positive genetic test for $\mathrm{FH}$, only a small percentage was categorized as definite $\mathrm{FH}$, in line with previous observations reporting that these criteria are not valid in children, whereas other criteria might be more appropriate as they contain specific cut- 
Table 3

DLCN score by missing information.

\begin{tabular}{|c|c|c|c|c|}
\hline \multirow[t]{2}{*}{ Number of missing } & \multicolumn{4}{|l|}{ DLCN score } \\
\hline & Unlikely (0-2) & Possible (3-5) & Probable (6-8) & Definite $(>8)$ \\
\hline 0 missing & $5.01 \%$ & $29.53 \%$ & $30.55 \%$ & $34.92 \%$ \\
\hline At least 1 missing in Group 1 (family history) & $5.89 \%$ & $26.79 \%$ & $25.89 \%$ & $41.43 \%$ \\
\hline At least 1 missing in Group 2 (clinical history) & $9.79 \%$ & $54.55 \%$ & $24.48 \%$ & $11.19 \%$ \\
\hline At least 1 missing in Group 3 (physical examination) & $9.04 \%$ & $40.36 \%$ & $21.08 \%$ & $29.52 \%$ \\
\hline
\end{tabular}

*Group reference Supplementary table 1.

Table 4

LDL-C levels and DLCN score in the mutation-positive sample based on sources of LDL-C levels.

Total FH population 1377 Not on statin $(\mathrm{N}=768)$ On statin treatment, with known pre-treatment LDL-C levels $(\mathrm{N}=397)$
On statin treatment, with estimated pre-treatment LDL-C levels $(\mathrm{N}=212)$

\begin{tabular}{|c|c|c|c|c|}
\hline \multicolumn{5}{|l|}{ LDL-C levels } \\
\hline$<155 \mathrm{mg} / \mathrm{dL}$ & $2.47 \%$ & $2.60 \%$ & $1.26 \%$ & $4.25 \%$ \\
\hline $155-190 \mathrm{mg} / \mathrm{dL}$ & $6.46 \%$ & $7.42 \%$ & $6.30 \%$ & $3.30 \%$ \\
\hline $191-250 \mathrm{mg} / \mathrm{dL}$ & $28.40 \%$ & $28.91 \%$ & $31.74 \%$ & $20.28 \%$ \\
\hline $251-325 \mathrm{mg} / \mathrm{dL}$ & $36.75 \%$ & $39.06 \%$ & $34.51 \%$ & $32.55 \%$ \\
\hline$>325 \mathrm{mg} / \mathrm{dL}$ & $25.93 \%$ & $22.01 \%$ & $26.20 \%$ & $39.62 \%$ \\
\hline \multicolumn{5}{|l|}{ DLCN score } \\
\hline Definite $>8$ & $37.91 \%$ & $33.46 \%$ & $37.53 \%$ & $54.72 \%$ \\
\hline Probable 6-8 & $28.47 \%$ & $27.47 \%$ & $30.98 \%$ & $27.36 \%$ \\
\hline Possible 3-5 & $28.32 \%$ & $31.90 \%$ & $28.72 \%$ & $14.62 \%$ \\
\hline Unlikely $<3$ & $5.30 \%$ & $7.16 \%$ & $2.77 \%$ & $3.30 \%$ \\
\hline
\end{tabular}

off for LDL-C levels in this specific group [11].

From this analysis, it appears obvious that the correct application of the DLCN score requires that all the information included in the algorithm must be solicited by the physician during the patient visit, to avoid a misclassification and address the right subject to the genetic testing. It is worth noting, however, that the less severe phenotypes may not be classified as definite $\mathrm{FH}$, and on the other hand, a polygenic form of hypercholesterolemia might be not recognized during a genetic testing. We cannot exclude that the low performance of the DLCN score observed in our study could be related to the extension of the genetic analysis to the young relatives of the index patients, in which the suspect of the disease was suggested basically by lipid levels and by the presence of the mutation in the family. This identifies a group of subjects for whom opportunistic screening based on clinical algorithms would be ineffective, highlighting the decisive role of cascade screening.

Although the DLCN score is undoubtedly a very useful tool for the physician in the diagnosis of $\mathrm{FH}$, in daily practice it could be limited by difficulty in finding information; moreover, it failed to identify a third of the subjects with genetic diagnosis of FH. Even if an update of this tool and its validation in individual national contexts would be warranted, physicians should be aware that it is just a support tool and must rely on their clinical judgment.

\section{Conflicts of interest}

The authors declared they do not have anything to disclose regarding conflict of interest with respect to this manuscript.

\section{Author contributions}

MC, EO, and ALC were responsible for the study concept and design. EO did the analysis. MC and ALC contributed to the data analysis and interpretation of the results. MC and AP drafted the manuscript and all authors critically revised for important intellectual content and approved the final manuscript.

\section{Acknowledgments}

The work of ALC is supported by: Fondazione Cariplo 2015-0524 and 2015-0564; H2020 REPROGRAM PHC-03-2015/667837-2. The LIPIGEN study is an initiative of the SISA Foundation supported by an unconditional research grant from Sanofi.

\section{Appendix A. Supplementary data}

Supplementary data related to this article can be found at https://doi.org/ 10.1016/j.atherosclerosis.2018.08.013.

\section{References}

[1] B.G. Nordestgaard, M.J. Chapman, S.E. Humphries, et al., Familial hypercholesterolaemia is underdiagnosed and undertreated in the general population: guidance for clinicians to prevent coronary heart disease: consensus statement of the European atherosclerosis society, Eur. Heart J. 34 (2013) 3478-3490a.

[2] P.J. Talmud, S. Shah, R. Whittall, et al., Use of low-density lipoprotein cholesterol gene score to distinguish patients with polygenic and monogenic familial hypercholesterolaemia: a case-control study, Lancet 381 (2013) 1293-1301.

[3] World Health Organization, Human Genetics Programme. Familial Hypercholesterolemia: Report of a Second WHO Consultation, WHO, Geneva, 1999 WHO/HGN/FH/Cons/99.2.

[4] Risk of fatal coronary heart disease in familial hypercholesterolaemia. Scientific Steering Committee on behalf of the Simon Broome Register Group, BMJ 303 (1991) 893-896.

[5] J.C. Defesche, P.J. Lansberg, M.A. Umans-Eckenhausen, et al., Advanced method for the identification of patients with inherited hypercholesterolemia, Semin. Vasc. Med. 4 (2004) 59-65.

[6] M. Averna, A.B. Cefalu, M. Casula, et al., Familial hypercholesterolemia: the Italian atherosclerosis society network (LIPIGEN), Atherosclerosis Suppl. 29 (2017) 11-16.

[7] J. Besseling, I. Kindt, M. Hof, et al., Severe heterozygous familial hypercholesterolemia and risk for cardiovascular disease: a study of a cohort of 14,000 mutation carriers, Atherosclerosis 233 (2014) 219-223.

[8] M. Benn, G.F. Watts, A. Tybjaerg-Hansen, et al., Familial hypercholesterolemia in the Danish general population: prevalence, coronary artery disease, and cholesterol lowering medication, J. Clin. Endocrinol. Metabol. 97 (2012) 3956-3964.

[9] A.L. Catapano, I. Graham, G. De Backer, et al., 2016 ESC/EAS guidelines for the management of dyslipidaemias: the task force for the management of dyslipidaemias of the European society of cardiology (ESC) and European atherosclerosis society (EAS) developed with the special contribution of the European assocciation for cardiovascular prevention \& rehabilitation (EACPR), Atherosclerosis 253 (2016) 281-344.

[10] L. Troeung, D. Arnold-Reed, W. Chan She Ping-Delfos, et al., A new electronic screening tool for identifying risk of familial hypercholesterolaemia in general practice, Heart 102 (2016) 855-861.

[11] A. Wiegman, S.S. Gidding, G.F. Watts, et al., Familial hypercholesterolaemia in children and adolescents: gaining decades of life by optimizing detection and treatment, Eur. Heart J. 36 (2015) 2425-2437. 